\title{
The Hydrodynamic Nonlinear Schrödinger Equation: Space and Time
}

\author{
Amin Chabchoub ${ }^{1,2, t, *}$ and Roger H. J. Grimshaw ${ }^{3,+}$ \\ Graduate School of Frontier Sciences, The University of Tokyo, Chiba 277-8563, Japan \\ Department of Mechanical Engineering, Aalto University, Espoo 02150, Finland \\ 3 Department of Mathematics, University College London, London, WC1E 6BT, UK; r.grimshaw@ucl.ac.uk \\ * Correspondence: amin.chabchoub@isea.k.u-tokyo.ac.jp; Tel./Fax: +81-7136-4880 \\ $\dagger$ These authors contributed equally to this work.
}

Academic Editor: Pavel Berloff

Received: 11 April 2016; Accepted: 11 July 2016; Published: 19 July 2016

\begin{abstract}
The nonlinear Schrödinger equation (NLS) is a canonical evolution equation, which describes the dynamics of weakly nonlinear wave packets in time and space in a wide range of physical media, such as nonlinear optics, cold gases, plasmas and hydrodynamics. Due to its integrability, the NLS provides families of exact solutions describing the dynamics of localised structures which can be observed experimentally in applicable nonlinear and dispersive media of interest. Depending on the co-ordinate of wave propagation, it is known that the NLS can be either expressed as a space- or time-evolution equation. Here, we discuss and examine in detail the limitation of the first-order asymptotic equivalence between these forms of the water wave NLS. In particular, we show that the the equivalence fails for specific periodic solutions. We will also emphasise the impact of the studies on application in geophysics and ocean engineering. We expect the results to stimulate similar studies for higher-order weakly nonlinear evolution equations and motivate numerical as well as experimental studies in nonlinear dispersive media.
\end{abstract}

Keywords: nonlinear waves; localized structures; rogue waves

\section{Introduction}

The theory of weakly nonlinear water waves has been found to be very useful for the modelling of ocean waves [1-3]. In finite and infinite water depth, the nonlinear Schrödinger equation (NLS) is the simplest evolution equation of this kind that takes into account dispersion and nonlinearity. Being an integrable equation, the NLS provides exact analytical solutions that describe the evolution of localised structures on the water surface in time and space, thus allowing subsequently the study and understanding of the dynamics of fundamental localised structures. The validity of the NLS has been experimentally confirmed even in the modelling of extreme localisations, beyond its well-known asymptotic limitations [4-8], and due to its interdisciplinary character analogies being able to be built into other nonlinear dispersive media, such as in optics [9], a research field in which several NLS applications have found strong interest [9-14]. Furthermore, the NLS admits basic models for the description of oceanic extreme events known as breathers [15-17]. Indeed, the family of Akhmediev breathers (ABs) [18] and Peregrine breathers [18,19] are strongly connected to the modulation instability (MI), also known as Benjamin-Feir instability [20], of Stokes waves [21]. In fact, the experimental investigation of exact solutions of the NLS, either numerically or in water wave facilities, has increased the degree of understanding of nonlinear and unstable water waves, as well as allowing the characterisation of the limitations of weakly nonlinear hydrodynamic models [8]. The choice of choosing the NLS representation of wave evolution in either time or space depends on the type of investigation, that is, depending on the space or time evolution coordinate of interest. 
Here, we investigate the asymptotic equivalence of both possible evolution expressions of the NLS within the context of exact solutions, ranging from stationary localised to pulsating and unstable envelopes. We show that, for a particular family of periodic solutions, such as it is the case for $\mathrm{ABs}$, the latter equivalence is not valid. A detailed analysis of this feature, and its consequences and potential applications, will be discussed.

\section{Analysis}

We will first discuss the general propagation of wave packets with respect to either the space- or time-NLS, describing the asymptotic equivalence or otherwise, illustrating the mismatch of the localised wave propagation of exact NLS solutions, dimensionalized to satisfy both forms of the NLS in the spatio-temporal physical plane.

\subsection{The Propagation of Wave Packets in Time and Space}

In deep water, the spatio-temporal surface elevation $\zeta(x, t)$ is given by, at leading order, as one of the two expressions,

$$
\begin{aligned}
& \text { Case A : } \quad \zeta(x, t)=\frac{1}{2}[A(x, t) \exp (i \vartheta)+\text { c. c. }+\cdots], \\
& \text { Case B : } \quad \zeta(x, t)=\frac{1}{2}[B(x, t) \exp (i \vartheta)+\text { c. c. }+\cdots] .
\end{aligned}
$$

Here, c. c. denotes the complex conjugate, and $A(x, t), B(x, t)$ are complex wave amplitudes of $O(\alpha)$ that are slowly varying relative to the phase $\vartheta=k x-\omega t$. The omitted terms are $O\left(\alpha^{2}\right)$, and the derivatives of $A, B$ are also $O\left(\alpha^{2}\right)$. Later, we shall insert the small parameter $\alpha$ explicitly. The wave frequency $\omega$ and wavenumber $k$ are related through the linear dispersion relation $\omega=\sqrt{g k}$, where $g$ denotes the gravitational acceleration. At the leading order in a weakly nonlinear asymptotic expansion, $A$ satisfies the "space-NLS" equation [22]:

$$
\begin{gathered}
i\left(A_{t}+c_{g} A_{x}\right)+\lambda A_{x x}+\mu|A|^{2} A=0, \\
c_{g}=\frac{\partial \omega}{\partial k}=\frac{\omega}{2 k}, \quad \lambda=\frac{1}{2} \frac{\partial^{2} \omega}{\partial k^{2}}=-\frac{\omega}{8 k^{2}}, \quad \mu=-\frac{\omega k^{2}}{2},
\end{gathered}
$$

appropriate for an initial-value problem, which is set to be

$$
A(x, 0)=A_{0}(x)
$$

For a hydrodynamic wavemaker problem, $B$ satisfies the "time-NLS" equation [2]:

$$
\begin{gathered}
i\left(B_{x}+\frac{1}{c_{g}} B_{t}\right)+\delta B_{t t}+v|B|^{2} B=0, \\
\delta=\frac{\lambda}{c_{g}^{3}}=-\frac{1}{g} \quad v=\frac{\mu}{c_{g}}=-k^{3} .
\end{gathered}
$$

At the wavemaker, we impose the boundary condition

$$
B(0, t)=B_{0}(t)
$$

The "space-NLS" Equation (3) and the "time-NLS" Equation (6) are asymptotically equivalent up to $O\left(\alpha^{3}\right)$, but importantly are not identical. In each of the Equations (1) and (2), the first two terms are $O\left(\alpha^{2}\right)$, the remaining terms are $O\left(\alpha^{3}\right)$, and the omitted terms are $O\left(\alpha^{4}\right)$. At the leading linear 
non-dispersive order, $O\left(\alpha^{2}\right)$, the solution of Equations (3) and (5) is $A_{0}\left(x-c_{g} t\right)$, and the solution of Equations (6) and (8) is $B_{0}\left(t-x / c_{g}\right)$, since these must agree at this leading order,

$$
A_{0}(x) \sim B_{0}\left(-x / c_{g}\right), \quad B_{0}(t) \sim A_{0}\left(-c_{g} t\right) .
$$

Each of these distinct NLS equations can be cast into the same canonical form. Thus, in case A, we set

$$
\begin{aligned}
& A=\frac{Q^{*}}{k}, \quad X=K\left(x-c_{g} t\right), \quad T=\Omega t, \\
& K=\sqrt{\frac{\Omega}{|\lambda|}}=\sqrt{2} k, \quad \Omega=-\frac{\mu}{2 k^{2}}=\frac{\omega}{4},
\end{aligned}
$$

where * denotes the complex conjugate. Then, Equation (3) takes the canonical form, in non-dimensional variables,

$$
i Q_{T}+Q_{X X}+2|Q|^{2} Q=0 .
$$

On the other hand, in case B, we set

$$
B=\frac{Q^{*}}{k}, \quad X=-K\left(x-c_{g} t\right), \quad T=\frac{\Omega x}{c_{g}} .
$$

It is useful to note that $\Omega / c_{g}=k / 2$. The outcome is again the canonical NLS Equation (12). The transformations are identical, except that, in case $\mathrm{A}, T$ transforms to $t$ but in case $\mathrm{B}, \mathrm{T}$ transforms to $x$. This difference may be significant as we now show.

Each solution $Q(X, T)$ of the canonical Equation (12) generates a corresponding solution of either Equation (3) through the transformation Equation (10), or Equation (6) through the transformation Equation (13). The corresponding initial-value problem Equation (9) or wavemaker condition Equation (8) is obtained by putting $T=0$ in each case. Although the outcome is different, one might expect that, nevertheless, the solutions in each case will be asymptotically equivalent.

This is the issue that we will now explore. It is important to note that for any solution $Q(X, T)$ of Equation (12), then $\alpha Q\left(\alpha x, \alpha^{2} t\right)$ is also a solution for any real parameter $\alpha>0$. We shall take solutions of Equation (12) in this form so that the small parameter $\alpha$ is explicitly displayed. Then, asymptotic equivalence is expected in the limit when the amplitude parameter $\alpha \rightarrow 0$. Thus, each solution of Equation (12) generates a solution of the "space-NLS" Equation (3) or the "time-NLS" Equation (6),

$$
\begin{gathered}
A=\alpha Q^{*}\left(\sqrt{2} \alpha k\left(x-c_{g} t\right), \frac{\alpha^{2} \omega t}{4}\right), \quad \alpha=k a, \\
B=\alpha Q^{*}\left(-\sqrt{2} \alpha k\left(x-c_{g} t\right), \frac{\alpha^{2} \omega x}{4 c_{g}}\right), \quad \alpha=k a,
\end{gathered}
$$

where $\alpha$ is the wave steepness. These clearly agree at the leading order, being identical in the dependence on the dominant phase variable $x-c_{g} t$. However, they differ in the slow $t$ and $x$ dependence, and are in general only asymptotically equivalent when $x-c_{g} t=O(\alpha)$. That is apparently only when the solutions are localised around the phase line $x=c_{g} t$. In practice, solutions are used for small, but finite non-zero $\alpha$ and an asymptotic equivalence in the two types of NLS dynamics is expected. Next, we will discuss analytically the significant differences that will arise due to the two different dimensional transformations, especially for solutions which have a particular localisation. A detailed description of this latter fact will be discussed and illustrated in the next section with several specific examples. 


\subsection{The Evolution of Specific NLS Solutions}

We will now discuss this equivalence or otherwise within the framework of some exact NLS solutions [15]. First, we consider the fundamental and stationary envelope soliton solution [23]:

$$
Q_{S}(X, T)=\alpha \operatorname{sech}(\alpha X) \exp \left(i \alpha^{2} T\right) .
$$

This is a family of solutions with the single parameter being the amplitude $\alpha$. Using the transformations Equations (14) or (15), respectively, we obtain the following two possible dimensional forms of this localised structure:

$$
\begin{aligned}
& A_{S}(x, t)=a \operatorname{sech}\left(\sqrt{2} \alpha k\left(x-c_{g} t\right)\right) \exp \left(-\frac{i \alpha^{2} \omega t}{4}\right), \\
& B_{S}(x, t)=a \operatorname{sech}\left(\sqrt{2} \alpha k\left(x-c_{g} t\right)\right) \exp \left(-\frac{i \alpha^{2} \omega x}{4 c_{g}}\right) .
\end{aligned}
$$

Note again that $\alpha$ is the wave steepness and that $\omega / c_{g}=2 k$. These solutions differ only in the chirp factor, which, in case $\mathrm{A}$, is a nonlinear frequency correction $\Delta \omega=-\alpha^{2} \omega / 4$ to the linear frequency $\omega$, and, in case $\mathrm{B}$, is a nonlinear wavenumber correction $\Delta k=\alpha^{2} \omega / 4 c_{g}$ to the linear wavenumber $k$. However, these are asymptotically equivalent as from the linear dispersion relation $\Delta \omega+c_{g} \Delta k=0$. The spatio-temporal dynamics of the respective solutions $A, B$ from Equations (17) and (18) are plotted in Figure 1.
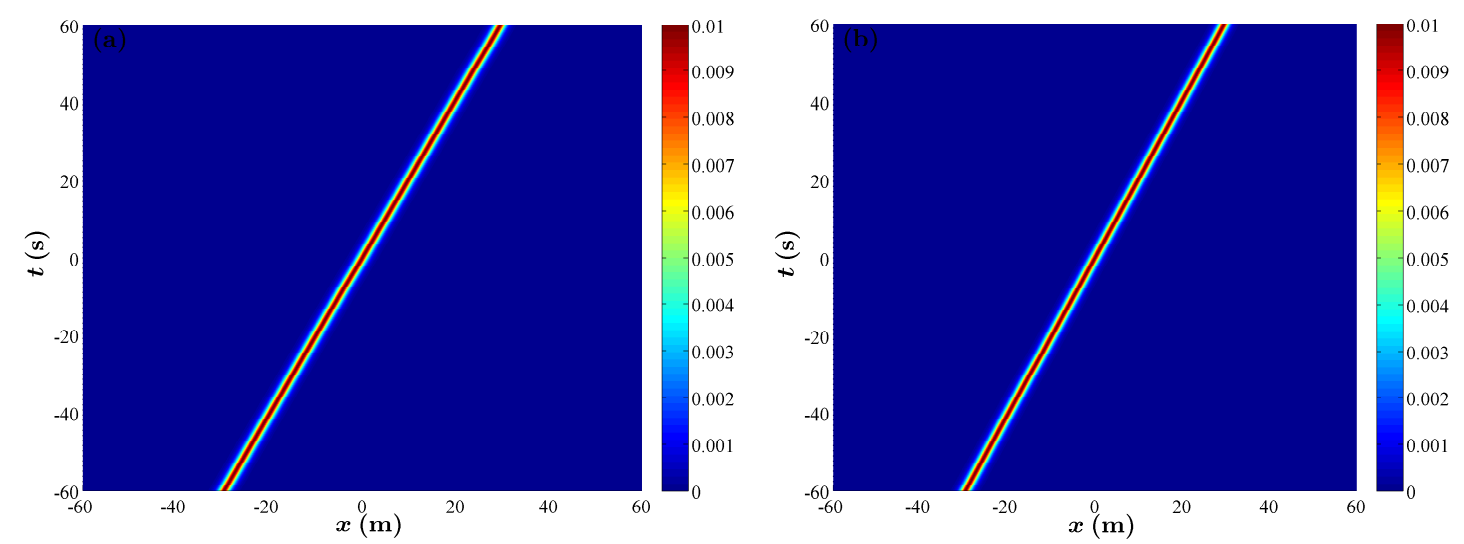

Figure 1. (a) spatiotemporal evolution of the modulus of the soliton $\left|A_{S}(x, t)\right|$ for the carrier parameters $a k=0.1$ and $a=0.01 \mathrm{~m}$; (b) spatiotemporal evolution of the modulus of the soliton $\left|B_{S}(x, t)\right|$ for the carrier parameters $a k=0.1$ and $a=0.01 \mathrm{~m}$.

Second, we consider the doubly-localised Peregrine breather [19]:

$$
Q_{P}(X, T)=\alpha\left(-1+\frac{4\left(1+4 i \alpha^{2} T\right)}{1+4 \alpha^{2} X^{2}+16 \alpha^{4} T^{2}}\right) \exp \left(2 i \alpha^{2} T\right) .
$$

Again, using the transformations (14) or (15), respectively, we obtain

$$
\begin{gathered}
A_{P}(x, t)=a\left(-1+\frac{4\left(1-i \alpha^{2} \omega t\right)}{1+8 k^{2} \alpha^{2}\left(x-c_{g} t\right)^{2}+\omega^{2} \alpha^{4} t^{2}}\right) \exp \left(-\frac{i \omega \alpha^{2} t}{2}\right), \\
B_{P}(x, t)=a\left(-1+\frac{4\left(1-i \omega \alpha^{2} x / c_{g}\right)}{1+8 k^{2} \alpha^{2}\left(x-c_{g} t\right)^{2}+\omega^{2} \alpha^{4} x^{2} / c_{g}^{2}}\right) \exp \left(-\frac{i \omega \alpha^{2} x}{2 c_{g}}\right) .
\end{gathered}
$$

Although these solutions are different both in the amplitude and in the chirp factor, they are asymptotically equivalent in the limit $\alpha \rightarrow 0$, as when $x-c_{g} t=O(\alpha)$, they are almost identical, 
and agree completely to within terms of $\mathcal{O}\left(\alpha^{4}\right)$. Importantly, the solutions are localised along $x=c_{g} t$. The respective solutions $A, B$ from Equations (20) and (21) are depicted in Figure 2.
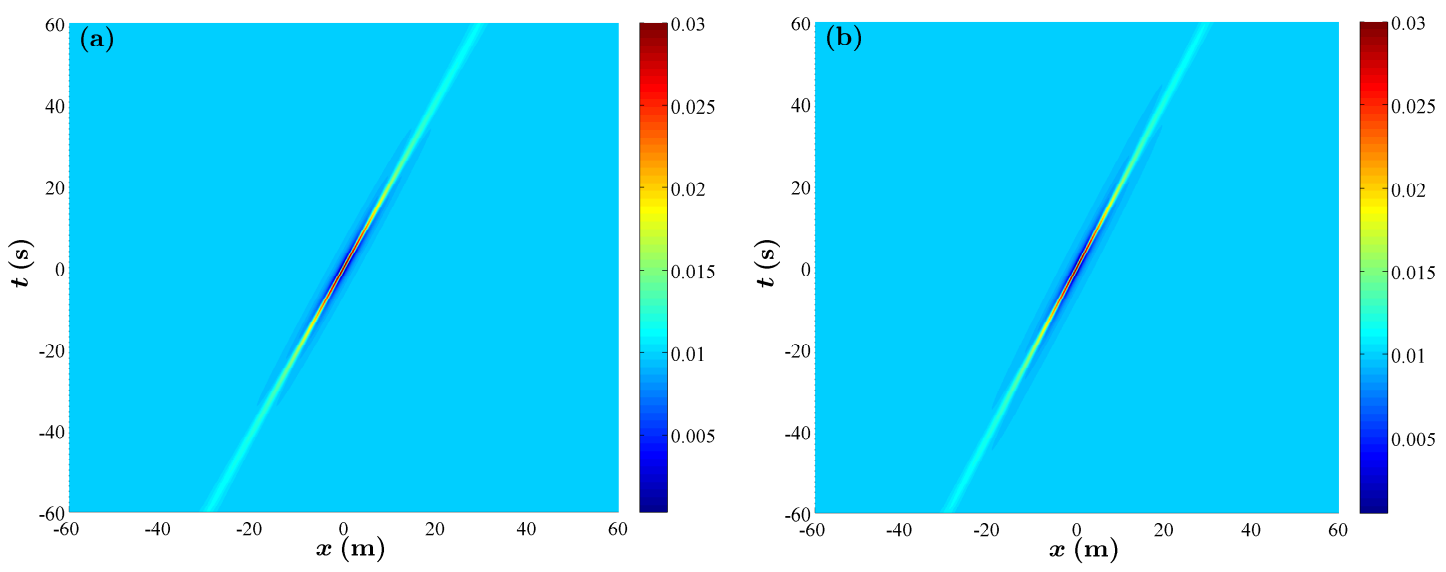

Figure 2. (a) spatiotemporal evolution of the modulus of the Peregrine breather $\left|A_{P}(x, t)\right|$ for the carrier parameters $a k=0.1$ and $a=0.01 \mathrm{~m}$; (b) spatiotemporal evolution of the modulus of the Peregrine breather $\left|B_{P}(x, t)\right|$ for the carrier parameters $a k=0.1$ and $a=0.01 \mathrm{~m}$.

Next, we consider the periodic Kuznetsov-Ma breather [24,25]

$$
Q_{K M}(X, T)=\alpha\left(\frac{\cos \left(\Lambda \alpha^{2} T-2 i \varphi\right)-\cosh (\varphi) \cosh (p \alpha X)}{\cos \left(\Lambda \alpha^{2} T\right)-\cosh (\varphi) \cosh (p \alpha X)}\right) \exp \left(2 i \alpha^{2} T\right),
$$

where $\Lambda=2 \sinh (2 \varphi), p=2 \sinh (\varphi)$ and $\varphi$ is a real-valued parameter. This is localised in $X$ and periodic in T. After applying the transformations Equations (14) or (15), respectively, it becomes

$$
\begin{aligned}
A_{K M}(x, t)= & a\left(\frac{\cos \left(\frac{\Lambda \alpha^{2} \omega t}{4}+2 i \varphi\right)-\cosh (\varphi) \cosh \left(p \sqrt{2} \alpha k\left(x-c_{g} t\right)\right)}{\cos \left(\frac{\Lambda \alpha^{2} \omega t}{4}\right)-\cosh (\varphi) \cosh \left(p \sqrt{2} \alpha k\left(x-c_{g} t\right)\right)}\right) \\
& \exp \left(-\frac{i \omega \alpha^{2} t}{2}\right), \\
B_{K M}(x, t)=a & \left(\frac{\cos \left(\frac{\Lambda \alpha^{2} \omega x}{4 c_{g}}+2 i \varphi\right)-\cosh (\varphi) \cosh \left(p \sqrt{2} \alpha k\left(x-c_{g} t\right)\right)}{\cos \left(\frac{\omega \Lambda \alpha^{2} x}{4 c_{g}}\right)-\cosh (\varphi) \cosh \left(p \sqrt{2} \alpha k\left(x-c_{g} t\right)\right)}\right) \\
& \exp \left(-\frac{i \omega \alpha^{2} x}{2 c_{g}}\right) .
\end{aligned}
$$

Although formally different, each is localized in $x-c_{g} t$ and periodic in $t, x$, respectively. They are then asymptotically equivalent in the limit $\alpha \rightarrow 0$, as when $x-c_{g} t=O(\alpha)$, they are almost identical, and also agree completely to within terms of $O\left(\alpha^{4}\right)$. The respective forms of the solutions $A, B$ from Equations (26) and (27) are plotted in Figure 3. 

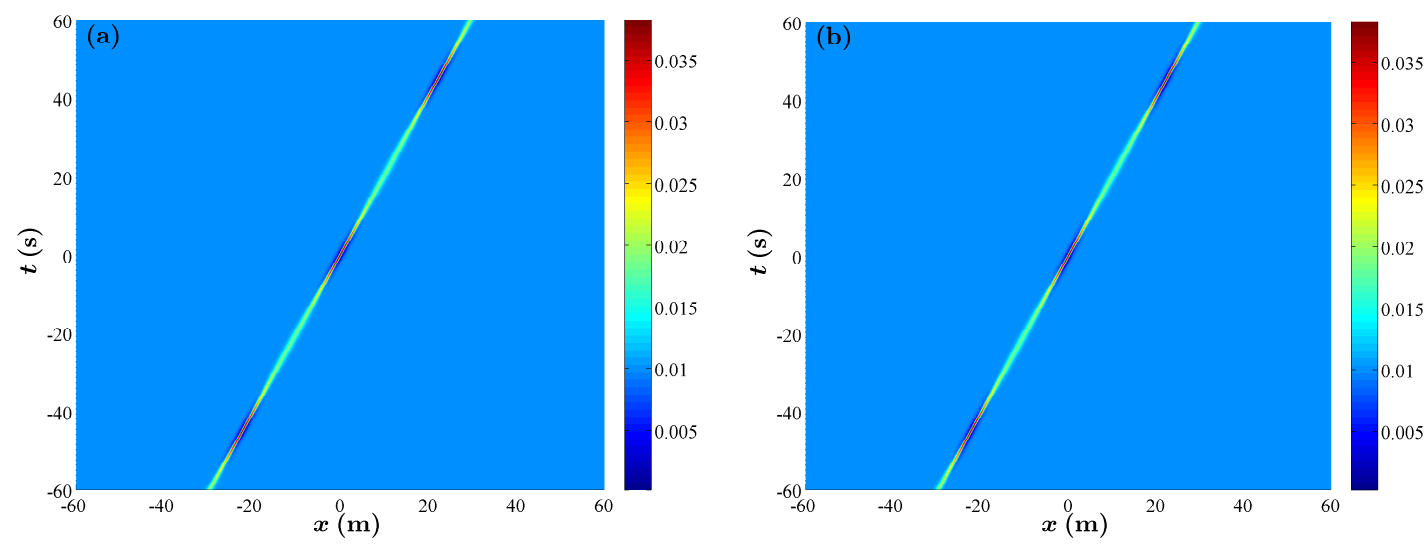

Figure 3. (a) spatiotemporal evolution of the modulus of an Kuznetsov-Ma breather $\left|A_{K M}(x, t)\right|$ for $\varphi=0.88$ and the carrier parameters $a k=0.1$ and $a=0.01 \mathrm{~m}$; (b) spatiotemporal evolution of the modulus of an Kuznetsov breather $\left|B_{K M}(x, t)\right|$ for $\varphi=0.88$ and the carrier parameters $a k=0.1$ and $a=0.01 \mathrm{~m}$.

Finally, we consider the family of Akhmediev breathers (ABs) $[18,26]$

$$
Q_{A}(X, T)=\alpha\left(\frac{\cosh \left(\Lambda \alpha^{2} T-2 i \varphi\right)-\cos (\varphi) \cos (p \alpha X)}{\cosh \left(\Lambda \alpha^{2} T\right)-\cos (\varphi) \cos (p \alpha X)}\right) \exp \left(2 i \alpha^{2} T\right)
$$

where $\Lambda=2 \sin (2 \varphi), p=2 \sin (\varphi)$ and $\varphi$ is a real-valued parameter. This is periodic in $X$ and localized in $T$. After transformation, as above, it becomes

$$
\begin{aligned}
A_{A}(x, t)= & a\left(\frac{\cosh \left(\frac{\Lambda \alpha^{2} \omega t}{4}+2 i \varphi\right)-\cos (\varphi) \cos \left(p \alpha \sqrt{2} \alpha k\left(x-c_{g} t\right)\right)}{\cosh \left(\frac{\Omega \alpha^{2} \omega t}{4}\right)-\cos (\varphi) \cos \left(p \alpha \sqrt{2} \alpha k\left(x-c_{g} t\right)\right)}\right) \\
& \exp \left(-\frac{i \omega \alpha^{2} t}{2}\right), \\
B_{A}(x, t)= & a\left(\frac{\cosh \left(\frac{\Lambda \alpha^{2} \omega x}{4 c_{g}}+2 i \varphi\right)-\cos (\varphi) \cos \left(p \sqrt{2} \alpha k\left(x-c_{g} t\right)\right)}{\cosh \left(\frac{\Omega \alpha^{2} \omega x}{4 c_{g}}\right)-\cos (\varphi) \cos \left(p \sqrt{2} \alpha k\left(x-c_{g} t\right)\right)}\right) \\
& \exp \left(-\frac{i \omega \alpha^{2} x}{2 c_{g}}\right) .
\end{aligned}
$$

Now, the case $\mathrm{A}$ and case $\mathrm{B}$ solutions are quite different. The respective solutions $A, B$ from Equations (26) and (27) are plotted in Figure 4.

The explanation is that while both the $A, B$ breathers are periodic in $x-c_{g} t$, the $A$-breather is localized in $t$ and the $B$-breather is localized in $x$. Hence, it is no longer possible to follow the solutions along the path where $x-c_{g} t=O(\alpha)$, and, therefore, establish asymptotic equivalence. Although, formally, the two solutions agree when $\alpha \rightarrow 0$, there is a persistent difference for small but finite $\alpha$. We conclude that these two breathers are two distinct solutions in the physical space. This is reinforced by noting that to observe an $A$-breather in a wave tank, one should record a time series at a fixed location, but to observe a $B$-breather, this will not reveal the breather motion at 
the wavemaker $x=0$, and, instead, one should take a snapshot at a fixed time and vice versa [27]. This fact is illustrated at the expected maximal breather focusing at $x=0$ in Figure 5.
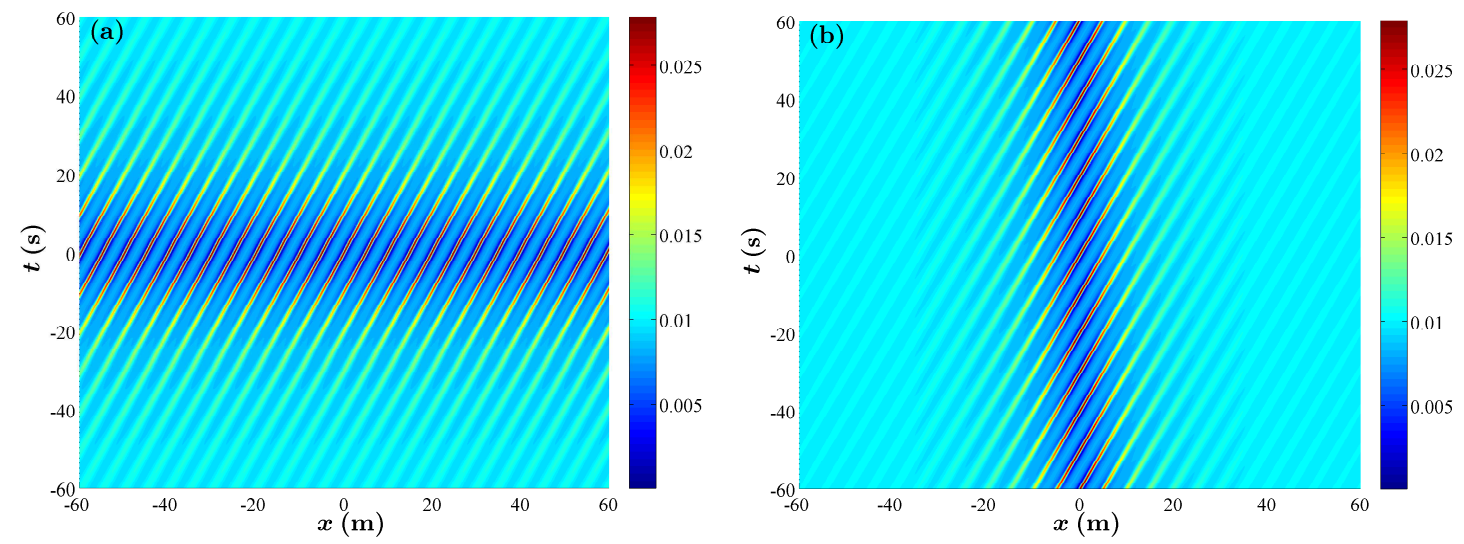

Figure 4. (a) spatiotemporal evolution of the modulus of an $\mathrm{AB}\left|A_{A}(x, t)\right|$ for $\varphi=0.46$ and the carrier parameters $a k=0.1$ and $a=0.01 \mathrm{~m}$; (b) spatiotemporal evolution of the modulus of an $\mathrm{AB}\left|B_{A}(x, t)\right|$ for $\varphi=0.46$ and the carrier parameters $a k=0.1$ and $a=0.01 \mathrm{~m}$.
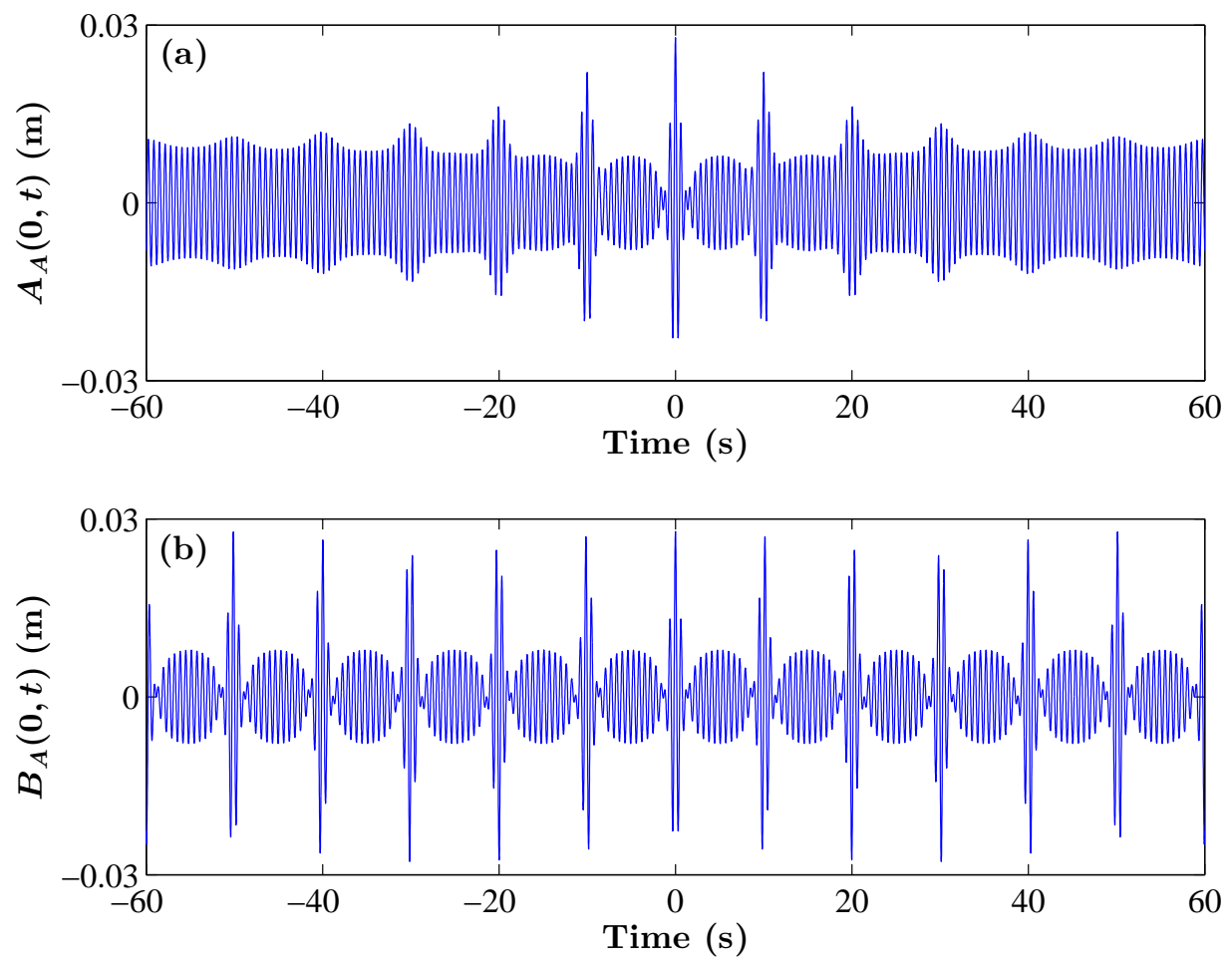

Figure 5. (a) temporal surface variation of an $\mathrm{AB} A_{A}(0, t)$ for $\varphi=0.46$ and background parameters $\alpha=0.1$ and $a=0.01 \mathrm{~m} ;(\mathbf{b})$ temporal water surface variation of an $\mathrm{AB} B_{A}(0, t)$ for $\varphi=0.46$ and background parameters $\alpha=0.1$ and $a=0.01 \mathrm{~m}$.

Note that the evolution of the ABs for the A case have been reported in [28]. In the latter work, the boundary conditions, applied to the wave maker, define a gradual amplification of the periodic AB-type wave field. The boundary conditions, with respect to the case B, correspond to a slight homogeneous periodic perturbation of the constant background. The case B has been measured in $[29,30]$. We also would like to point out that the classical MI problem [20,31], starting from a three wave system, can be only described by ABs of case B. The temporal spectra of the case A when very 
large positive and negative time scales are considered would almost remain stationary, when the wave train is evolving in space.

\section{Discussion and Conclusions}

To conclude, we have discussed the asymptotic equivalence of localised structures within the framework of either the space- or the time-NLS. The choice of one of these two configurations of the NLS depends on whether one is interested in integrating the evolution of wave packets in time (as for numerical simulations), or in space (as for experimental purposes by tracking the evolution of waves along a water wave facility). We have the validity of this equivalence or otherwise by means of some exact solutions of the NLS. When the solutions exhibit a symmetry in the space coordinate with respect to Equation (12), as it is the case for Akhmediev breathers (ABs), the equivalence fails. Thus, it is physically possible to generate two types of $A B$ solutions. This may be significant for applications. Indeed, breathers are nowadays used for several types of ocean engineering applications [32,33]. Generating ABs in a wave flume with respect to the space NLS allows for successive but progressive rogue waves gaining in amplitude with each impact at a fixed position, whereas with respect to the time-NLS, the solution would generate several extreme waves with similar envelope amplitudes in time, having different wave phases (and, therefore, different velocity fields), at the same position. When placing a model ship or offshore structure at the position of expected maximal $\mathrm{AB}$ compression, one could either study the wave impact related to progressive extreme events in the first case or to several rogue waves with similar significant heights in the second case. Indeed, it is known that deviations with respect to NLS dynamics are expected in an experiment, when the steepness of the carrier and the breather amplitude amplification are significant [8,34,35]. In fact, higher-order evolution equations such as the modified NLS [36-38], also known as Dysthe equations, or others [39-41] provide an accurate correction and a better prediction of the amplified wave field. These discrepancies with respect to NLS dynamics have an influence in the decay dynamics of the breathers after reaching the saturation point and a recurrence, referred to as Fermi-Pasta Ulam recurrence, is expected to occur [10,31,42-44]. We have also discussed the fact that, when considering the limiting case of infinite modulation period, which corresponds to the case of a Peregrine breather, both evolution dynamical forms become identical again in the dimensional spatio-temporal plane. We also expect these results to stimulate studies and applications in other nonlinear media such as in optics, Bose-Einstein Condensates, plasma and solids.

Acknowledgments: Amin Chabchoub is thankful to Odin Gramstad, Miguel Onorato and Nail Akhmediev for enlightening discussions. Amin Chabchoub acknowledges support from the Burgundy Region, the Association of German Engineers (VDI) and the Japan Society for the Promotion of Science (JSPS).

Author Contributions: Amin Chabchoub and Roger H. J. Grimshaw conducted the theoretical analysis, interpretation of the results and the writing of the manuscript equally.

Conflicts of Interest: The authors declare no conflict of interest.

\section{Abbreviations}

The following abbreviations are used in this manuscript:

NLS: Nonlinear Schrödinger equation

MI: Modulation instability

AB: Akhmediev breather

\section{References}

1. Kharif, C.; Pelinovsky, E.; Slunyaev, A. Rogue Waves in the Ocean; Springer: Berlin, Germany, 2009.

2. Osborne, A. Nonlinear Ocean Waves \& the Inverse Scattering Transform; Academic Press: Amsterdam, The Netherlands, 2010; Volume 97. 
3. Babanin, A. Breaking and Dissipation of Ocean Surface Waves; Cambridge University Press: Cambridge, UK, 2011.

4. Yuen, H.C.; Lake, B.M. Nonlinear deep water waves: Theory and experiment. Phys. Fluids (1958-1988) 1975, 18, 956-960.

5. Chabchoub, A.; Hoffmann, N.; Akhmediev, N. Rogue wave observation in a water wave tank. Phys. Rev. Lett. 2011, 106, 204502.

6. Toffoli, A.; Waseda, T.; Houtani, H.; Kinoshita, T.; Collins, K.; Proment, D.; Onorato, M. Excitation of rogue waves in a variable medium: An experimental study on the interaction of water waves and currents. Phys. Rev. E 2013, 87, 051201.

7. Slunyaev, A.; Clauss, G.F.; Klein, M.; Onorato, M. Simulations and experiments of short intense envelope solitons of surface water waves. Phys. Fluids (1994-present) 2013, 25, 067105.

8. Chabchoub, A.; Onorato, M.; Akhmediev, N. Hydrodynamic solitons and breathers. Rogue and Shock Waves; Onorato, M., Residori, S., Baronio, F., Eds.; Lecture Notes in Physics; Springer: Berlin, Germany, 2016.

9. Chabchoub, A.; Kibler, B.; Finot, C.; Millot, G.; Onorato, M.; Dudley, J.M.; Babanin, A.V. The nonlinear Schrödinger equation and the propagation of weakly nonlinear waves in optical fibers and on the water surface. Ann. Phys. 2015, 361, 490-500.

10. Dudley, J.M.; Genty, G.; Dias, F.; Kibler, B.; Akhmediev, N. Modulation instability, akhmediev breathers and continuous wave supercontinuum generation. Opt. Express 2009, 17, 21497-21508.

11. Kibler, B.; Fatome, J.; Finot, C.; Millot, G.; Dias, F.; Genty, G.; Akhmediev, N.; Dudley, J.M. The Peregrine soliton in nonlinear fibre optics. Nat. Phys. 2010, 6, 790-795.

12. Onorato, M.; Residori, S.; Bortolozzo, U.; Montina, A.; Arecchi, F.T. Rogue waves and their generating mechanisms in different physical contexts. Phys. Rep. 2013, 528, 47-89.

13. Dudley, J.M.; Dias, F.; Erkintalo, M.; Genty, G. Instabilities, breathers and rogue waves in optics. Nat. Photonics 2014, 8, 755-764.

14. Tlidi, M.; Gandica, Y.; Sonnino, G.; Averlant, E.; Panajotov, K. Self-Replicating spots in the brusselator model and extreme events in the one-dimensional case with delay. Entropy 2016, 18, 64, doi:10.3390/e18030064.

15. Dysthe, K.B.; Trulsen, K. Note on breather type solutions of the NLS as models for freak-waves. Phys. Scr. 1999, T82, 48-52.

16. Shrira, V.I.; Geogjaev, V.V. What makes the Peregrine soliton so special as a prototype of freak waves? J. Eng. Math. 2010, 67, 11-22.

17. Grimshaw, R.; Tovbis, A. Rogue waves: Analytical predictions. Proc. R. Soc. A 2013, 469, 20130094.

18. Akhmediev, N.; Eleonskii, V.M.; Kulagin, N.E. Generation of periodic trains of picosecond pulses in an optical fiber: Exact solutions. Sov. Phys. JETP 1985, 62, 894-899.

19. Peregrine, D.H. Water waves, nonlinear Schrödinger equations and their solutions. J. Aust. Math. Soc. Series B. Appl. Math. 1983, 25, 16-43.

20. Benjamin, T.B.; Feir, J. The disintegration of wave trains on deep water Part 1. Theory. J. Fluid Mech. 1967, $27,417-430$.

21. Stokes, G.G. On the theory of oscillatory waves. Trans. Camb. Philos. Soc. 1847, 8, 441-473.

22. Zakharov, V.E. Stability of periodic waves of finite amplitude on the surface of a deep fluid. J. Appl. Mech. Techn. Phys. 1968, 9, 190-194.

23. Shabat, A.; Zakharov, V. Exact theory of two-dimensional self-focusing and one-dimensional self-modulation of waves in nonlinear media. Sov. Phys. JETP 1972, 34, 62.

24. Kuznetsov, E. Solitons in a parametrically unstable plasma. Akademiia Nauk SSSR Doklady 1977, 236, 575-577.

25. Ma, Y.C. The perturbed plane-wave solutions of the cubic Schrödinger equation. Stud. Appl. Math. 1979, $60,43-58$.

26. Akhmediev, N.; Eleonskii, V.M.; Kulagin, N.E. Exact solutions of the first order of nonlinear Schrödinger equation. Theor. Math. Phys. (USSR) 1987, 72, 809-818.

27. Andonwati, Karjanto, N.; Van Groesen, E. Extreme wave phenomena in down-stream running modulated waves. Appl. Math. Model. 2007, 31, 1425-1443.

28. Chabchoub, A.; Kibler, B.; Dudley, J.; Akhmediev, N. Hydrodynamics of periodic breathers. Philos. Trans. A Math. Phys. Eng. Sci. 2014, 372, 20140005. 
29. Karjanto, N.; Van Groesen, E. Qualitative comparisons of experimental results on deterministic freak wave generation based on modulational instability. J. Hydro-Environ. Res. 2010, 3, 186-192.

30. Chabchoub, A.; Waseda, T. Hydrodynamic breathers modeling rogue waves. Proc. Violent Flows 2016, in press.

31. Tulin, M.P.; Waseda, T. Laboratory observations of wave group evolution, including breaking effects. J. Fluid Mech. 1999, 378, 197-232.

32. Onorato, M.; Proment, D.; Clauss, G.; Klein, M. Rogue waves: From nonlinear Schrödinger breather solutions to sea-keeping test. PLOS ONE 2013, 8, e54629.

33. Alberello, A.; Chabchoub, A.; Babanin, A.V.; Monty, J.M.; Elsnab, J.; Lee, J.H.; Bitner-Gregersen, E.M.; Toffoli, A. The veolicty field underneath linear and nonlinear breaking waves. In Proceedings of the ASME 2016 30th International Conference on Ocean, Offshore and Arctic Engineering, Busan, Korea, 19-24 June 2016; p. 54481.

34. Trulsen, K.; Dysthe, K.B. A modified nonlinear Schrödinger equation for broader bandwidth gravity waves on deep water. Wave Motion 1996, 24, 281-289.

35. Slunyaev, A.V.; Shrira, V.I. On the highest non-breaking wave in a group: Fully nonlinear water wave breathers versus weakly nonlinear theory. J. Fluid Mech. 2013, 735, 203-248.

36. Dysthe, K.B. Note on a modification to the nonlinear Schrödinger equation for application to deep water waves. Proc. R. Soc. Lond. A Math. Phys. Eng. Sci. 1979, 369, 105-114.

37. Trulsen, K.; Stansberg, C.T. Spatial evolution of water surface waves: Numerical simulation and experiment of bichromatic waves. In Proceedings of the Eleventh International Offshore and Polar Engineering Conference, Stavanger, Norway, 17-22 June 2001; International Society of Offshore and Polar Engineers: Mountain View, CA, USA, 2001.

38. Chabchoub, A.; Hoffmann, N.; Onorato, M.; Genty, G.; Dudley, J.M.; Akhmediev, N. Hydrodynamic supercontinuum. Phys. Rev. Lett. 2013, 111, 054104.

39. Slunyaev, A. A high-order nonlinear envelope equation for gravity waves in finite-depth water. J. Exp. Theor. Phys. 2005, 101, 926-941.

40. Gramstad, O.; Trulsen, K. Hamiltonian form of the modified nonlinear Schrödinger equation for gravity waves on arbitrary depth. J. Fluid Mech. 2011, 670, 404-426.

41. Craig, W.; Guyenne, P.; Sulem, C. A Hamiltonian approach to nonlinear modulation of surface water waves. Wave Motion 2010, 47, 552-563.

42. Fermi, E.; Pasta, J.; Ulam, S. Studies of the Nonlinear Problems; Los Alamos Report LA-1940; Los Alamos Laboratory of the University of California: Los Alamos, NM, USA, 1955.

43. Onorato, M.; Vozella, L.; Proment, D.; Lvov, Y.V. Route to thermalization in the $\alpha$-Fermi-Pasta-Ulam system. Proc. Natl. Acad. Sci. USA 2015, 112, 4208-4213.

44. Kimmoun, O.; Hsu, H.C.; Branger, B.; Li, M.S.; Chen, Y.Y.; Kharif, C.; Onorato, M.; Kelleher, E.J.R.; Kibler, B.; Akhmediev, N.; et al. Modulation instability and phase-shifted Fermi-Pasta-Ulam recurrence. 2016, arXiv:1602.01604 [nlin.PS].

(C) 2016 by the authors; licensee MDPI, Basel, Switzerland. This article is an open access article distributed under the terms and conditions of the Creative Commons Attribution (CC-BY) license (http://creativecommons.org/licenses/by/4.0/). 